
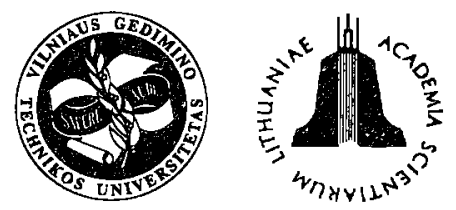

ISSN 1648-4142 TRANSPORT

http:/www.vtu.lt/english/editions

TRANSPORT - 2002, Vol XVII, No 3, 77-84

\title{
THE INVESTIGATION OF VIBRO-ISOLATING FEATURES OF TRANSMISSIONS OF VEHICLES IN ELASTIC CENTRIFUGAL COUPLINGS USING THE METHOD OF IDEN- TIFICATION OF DYNAMIC PROCESSES IN HALF-COUPLINGS ON THE UNIVERSAL DYNAMIC TEST STAND
}

\author{
Bronislovas Spruogis ${ }^{1}$, Leonas Zubavičius ${ }^{2}$ \\ ${ }^{1}$ Department of Transport Technology Equipment.E-mail: tti@ti.vtu.lt \\ ${ }^{2}$ Department of Printing Machines.E-mail: mechanic@me.vtu.lt \\ Vilnius Gediminas Technical University, Sauletekio al. 11, LT-2040 Vilnius, Lithuania
}

Received 20020201 ; accepted 20020418

\begin{abstract}
The presented paper describes the investigation of vibro-isolating features of transmissions of vehicles using universal dynamic test stand. The stand is protected by copyright and distinguishes itself for many advantages in comparison with the existing stands: it is contactless, precise in a wide frequency range, power-saving, high-speed. Due to low exploitation expenses and wide possibilities it is fit for the investigation of dynamic characteristics of various vehicles. On the said stand using the method of the identification of experiment dynamic processes, the investigation of vibroisolating features of rotor systems has been performed. The errors caused by the unevenness of rotation of connecting shafts, the adequacy of the calculated models and the dangerous resonance frequencies have been determined.
\end{abstract}

Keywords. Transmission, vibro-isolating features, an universal contactless stand, an elastic centrifugal coupling, the method of identification, a resonance frequency.

\section{Introduction}

Modern vehicles are being rapidly improved. New requirements related with the aspect of high-speed as well as the aspects of comfortability and safe exploitation are permanently made to them. High-speed of a vehicle causes special requirements for the structure of the vehicle. On the increase of speed of a vehicle the dynamic loads in kinematic couples of the vehicle increase as well. They are transferred to the transmission of the vehicle and its connecting elements. If the construction of the vehicle or any its elements are changed, they must be tested. For this purpose an experimental sample is made. Such experimental sample will be tested on the stand.

In the presented paper the investigation of vibroisolating features of transmissions of vehicles in elastic centrifugal couplings (ECC) using the method of the identification of dynamic processes in half-couplings on the universal dynamic test stand is developed by the authors [1-3]. Using the method of analytical identification [4 6], the investigation of vibro-isolating features of rotor systems has been performed, the errors caused by the unevenness of rotation of connecting shafts, the adequacy of calculated models and the dangerous resonance frequencies have been determined.

\section{The Review of the Stand}

Several methods and means for the measurement of dynamic characteristics of objects are known from technical references. They include photoelectric transformers of the moment of rotation, digital meters of the moment of rotation, phase dynamometers, stroboscopic meters of rotational vibrations, contactless electromagnetic sensors of the moment of rotation [7]. Their operation is based on the principle of magnetic anisotropy of ferro-magnetic substances.

All the above-mentioned methods have the same mutual imperfection: their carrying signal is of constant frequency, so it cannot be changed changing the rotational speed of the object under test. This imperfection reduces the possibilities of the test equipment: the range of usage becomes narrower, the accuracy becomes lower, the time of adjustment of the equipment grows and so on.

The stands of meters of a transferred moment of rotation [8] do not meet the requirements of today's technical progress.

The equipment of measurement of the moment of rotation [9] consists of an elastic element with measuring disks fixed on its ends and sensors of rotational speed of the elastic element. Each sensor is placed at the corresponding measuring disk and connected to inputs of 
the summator through its limiting amplifier. In the registering unit additional speed sensors with autonomous limiting amplifiers are installed; the angular shifts of the additional sensors are equal and opposite to the corresponding main sensors. The equipment also includes summators, integrators, comparators and so on.

However, the equipment [9] is not free of imperfection: it is not universal, its application is limited, prior preparation of the carrying signals is complicated and timeexpensive. So the exploitation expenses are considerable, it is impossible to change the carrying signal during a test, the accuracy of measurements is insufficient and so on. $[10,11]$.

The sphere of application of the test stand where the functions of electric brake of the load are performed by the electric generator connected to the power supply network is narrow, it is not universal and does not ensure an automatic control.

One of the mostly improved dynamic test stands is a set of tests and measurements of hydro-mechanical drives [12]. It consists of an internal combustion engine and an electric brake of load the output shafts of which are adapted to the connection of the cardan shafts of the hydro-mechanical drive under test. In addition, the disks with slits connected with pulse modulators are mounted on the output shafts. The modulators are connected to the measuring block. This set has the following disadvantages: the accuracy of measurements of objects under test is not ensured in a wide range of rotational speeds; additional time is needful for the calibration of the sensors; it is impossible to investigate a drive on a reverse; the universality (the analysis of co-varying functions as well as spectral densities and amplitude-frequency responses of dynamic objects) is not ensured because it is impossible to form an algorithm of the load of transitional processes.

\section{The Description of the Structural Scheme of the Origi- nal Stand}

Here we present the original universal dynamic test stand. Its construction is protected by copyright [1]. It is usable, for example, for the measurement of dynamic characteristics of couplings.

The universal dynamic test stand for the measurement of dynamic characteristics of couplings is shown in Fig 1. Magnetic disks 2 are fixed on the ends of the shafts of half-couplings of the coupling under test 1 . The shaft of one half-coupling is connected to the shaft of the driving engine 3 and the shaft of the second half-coupling with the shaft of the electric brake of the dynamic load 4 . The total shaft rotating system from the side of the driving engine ends with the upper extreme point sensor (UEP) with the tachogenerator 5 as well as two blocks of magnetic heads 6 and 7 , which inputs are connected to the block of the operating mode of the equipment 8 and the outputs - via the playback amplifiers 9 and 10 - to the two-channel amplifier 11 with automatic control.

One output of the amplifier 11 is connected to the input of the quantum pulse control generator 12 , the second output - to the meter of the coupling deformation angle and the transmitted moment of rotation 13 and the third output - to the spectral analyzer 14 . The output of the meter of the coupling deformation angle and the transmitted moment of rotation 13 is connected to the input of

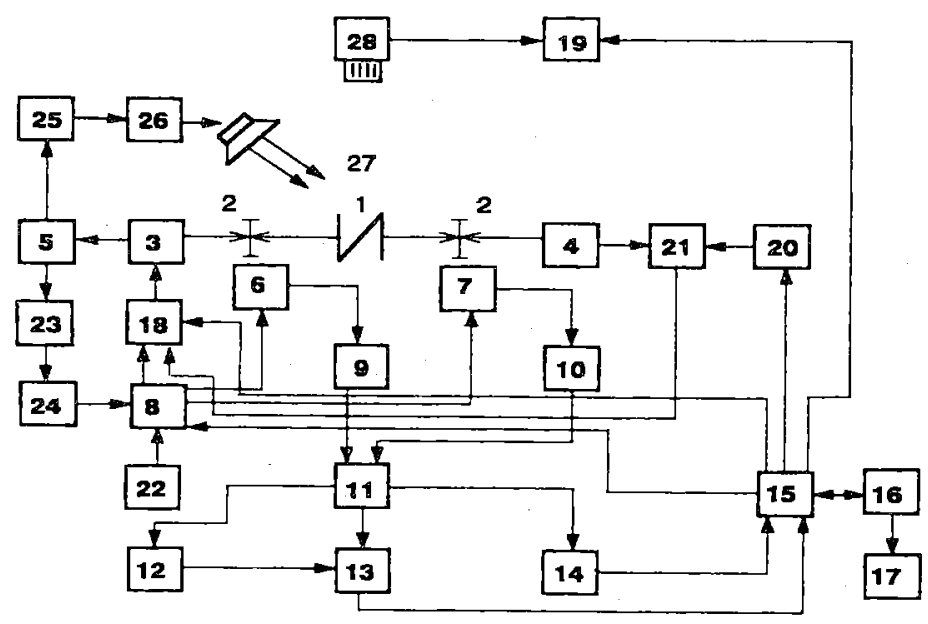

Fig 1. The structural scheme of the universal dynamic test stand: 1 - the coupling; 2 - magnetic disks; 3 - the driving engine; 4- the electric brake; 6,7 - the blocks of magnetic heads; 8 - the blocks of the operating mode; 9, 10 - the playback amplifiers; 11 - the two-channel amplifier; 12 - the generator; 13 - the meter of the moment of rotation; 14 - the spectral analyzer, 15 - the interface; 16 - the computer; 17 - the display; 18 - the control block of the driving engine; 19 - the control block of the video recorder; 20 - the control generator of the voltage of the load; 21 - the load control block; 22 - the magnetizing generator; 23 - the former of synchronous pulses; 24 - generator of cyclic frequency; 25 - the unit of formation of pulses of the upper extreme point; 26 - the pulse lamp control block; 27 - the pulse lamp; 28 - the video camera 
the interface 15 of the computer and the output of the spectral analyzer 14 is connected to the other input of the interface 15 of the computer. The first output of the interface is connected with the computer 16 , and the output of the computer is connected with the display 17. The test programme is transmitted from the computer 16 through four channels of the interface 16 : to the block of operating mode 8 , to the driving engine control block 18 , to the video recorder control block 19 and to the load control generator 20. The load control generator is connected with the load control block 21 . The voltage generated in the load generator 4 is transmitted to the load control block 21 ; its output is connected with the driving engine control block 18 according to the scheme of recuperation and the output of the latter is connected to the driving engine 3 . The shaft of the driving engine rotates the total system of the equipment in the direction of the coupling 1 , including the tachogenerator 5 which first output is connected with the input of the cyclic frequency generator control block through the amplifier-former of synchronous signals 23 , and the output of the said generator is connected to one input of the operating mode block 8 . The output of the upper extreme point (UEP) sensor of the tachogenerator 5 is connected to the UEP pulse forming unit 25 which output is connected to the input of the pulse lamp control block 26. The pulse lamp switching-on control signal from the output of the block 26 is transmitted to the pulse lamp 27. The useful video signal of the assembling unit upon our interest or the point of the coupling under test is transferred to the video camera 28 , and its output signal is applied to the input of the recorder 19. The latter gets the control signal from the interface 15 .

\section{The Principle of Operation of the Stand}

The universal dynamic test stand operates as follows.

The computer 16 via interface 15 sets the operating mode of the driving engine 3 with the control block 18 according to the preset programme. The magnetic disk 2 is fixed on one end of the shaft of the driving engine 3 and the end of the shaft is connected via the coupling 1 to the dynamic load 4; the same disk 2 is fixed on the shaft of the load. The mode of the dynamic load is preset by the control block 21 that receives control signals from the voltage control generator (VCG) 20. The mode of VCG is set via interface 15 according to the programme of the computer 16. The tachogenerator 5 with the upper extreme point (UEP) sensor is fixed on the other end of the shaft of the driving engine 3. UEP pulse forming unit 25 singles out and forms UEP pulse that controls the flash of the pulse lamp 27. Thus, the video camera shoots a film of rotation of the same point of the coupling 1. The tachogenerator 5 generates synchronous signals that are amplified in the amplifier - former of synchronous signals 23 and used for the synchronization of the cyclic frequency generator 24 .
The cyclic frequency from the generator is transmitted to the operating mode block where this frequency is mixed up with the frequency of the magnetizing generator 22 . From the operating mode block, the cyclic frequency via magnetized signal is transmitted to the magnetic heads 6 and 7 . Then the carrying cyclic frequency signal is recorded on the magnetic disks 2 . Rotation of the driving engine 3 is changeable according to the changes of the programme of the computer 16. Dependently on the direction of the operating mode, the signal for the control of the blocks of magnetic heads 6 and 7 is formed. Each block of magnetic heads 6 and 7 contains 5 magnetic heads: one erasing head, two recording heads and two playing heads.

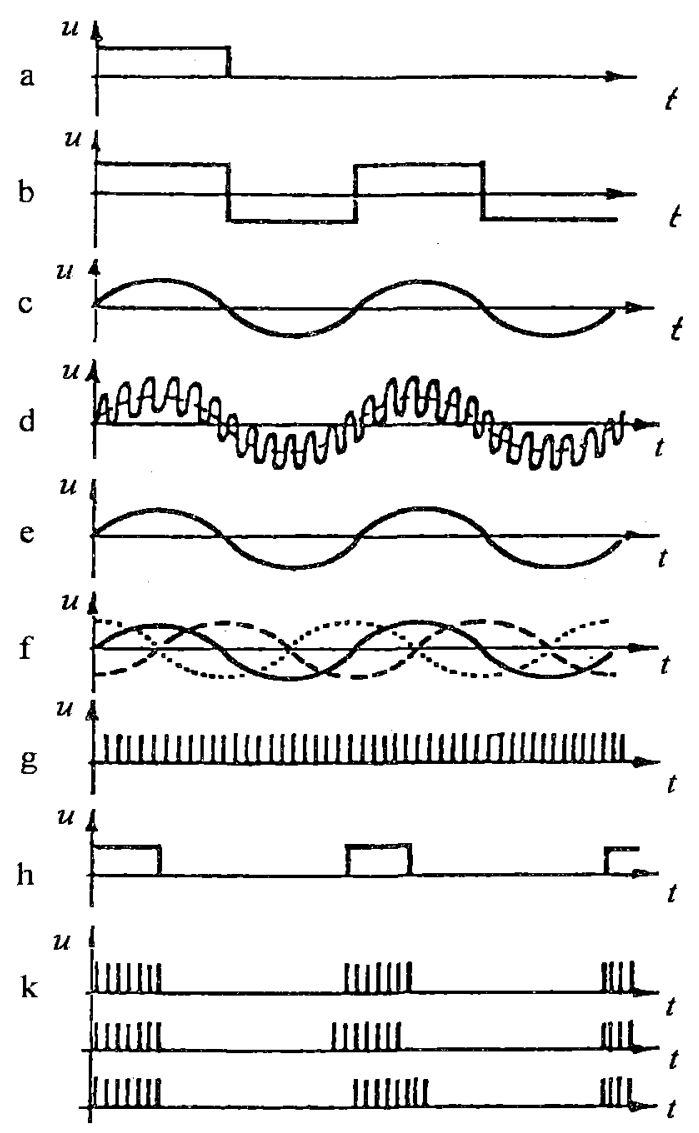

Fig 2. The oscillograms illustrating the principle of measurement of the coupling deformation angle using the universal dynamic test stand: $a-a$ pulse from UEP; $b$ - a pulse from the tachogenerator; $c$ - the cycle generator; $d$ - recording, when $\mathrm{f}_{\text {magn }}>5 \mathrm{f}_{\text {max.tak. }}\left(\mathrm{f}_{\text {magn }}-\right.$ the magnetizing frequency, $5 \mathrm{f}_{\text {max.tak. }}$ - the maximal cyclic frequency); $e$ - the signal of the playback of the block of magnetic heads 6; $f$ - the signal of the playback of the block of magnetic heads 7: - - when the deformation angle is equal to zero, - - when the deformation angle is positive; ..........- when the deformation angle is negative; $g$ - pulses of cyclic frequency generator $24: \mathrm{f}_{\text {tax. }} / \mathrm{f}_{\text {batg. }}=$ const; where $\mathrm{f}_{\text {6atg }}$ - the playback signals of the block of magnetic heads $6 ; \mathrm{h}-$ the phase shift of the playback signals of the blocks of magnetic heads 6 and $7 ; \mathrm{k}-$ the output from the meter of the moment of rotation 13 to the computer 
According to the direction of rotation the mutual erasing head, one recording head and one playing head are switched on. When the direction is changed, the opposite heads are switched on. Both the blocks of magnetic heads operate in the analogous way.

The blocks of magnetic heads record the cyclic frequency on the magnetic disks 2 . The signals are played by the playing heads and transmitted to the primary playback amplifiers 9 and 10 . The amplified signals pass the amplifier 11 with automatic control where the signals are amplified to the same level.

Meter 13 of the deformation angle of the coupling 1 compares the phases of the sinusoidal signals played back by the blocks of magnetic heads 6 and 7 and the deformation angle of the coupling is found according to the phase shift. In absence of the coupling deformation, the phase signals are the same and the phase shift is absent. The principle of measurement of the phase shift is shown in the oscillograms (Fig 2). The phase shift is fixed by stroboscopic pulses from the quantum pulse generator 12 . The synchronization of quantum pulses according to the frequency and phase is performed according to the cyclic frequency signal played back by the block of magnetic head 6 . The signal determining the coupling rotation angle in a digital form is transmitted to the computer 16 via the interface 15. Information on extreme values of parameters is presented on the display 17 .

As it may be seen the presented universal dynamic test stand is contactless and its set performs the registration of parameters, their visual examination, processing of results and their fixation. It distinguishes itself for high accuracy in wide rotational frequency range, universality, economy, high-speed, low exploitation expenses and wide possibilities of the investigation of dynamic characteristics of various machines.

\section{Analytic Part}

All vibration-damping characteristics and other dynamical parameters of ECC are determined in the process going through the wide range of random vibrations. Rotational vibrations should be considered as the main dynamic process in the input of the first half-coupling and in the output of the second half-clutch. In order to obtain the data mentioned above bench experiments simulating the work of real ECC, are carried out [13-16].

The data of experiments are treated as the realization of real random events. In ECVM uninterruptible processes were recorded by a four channeled analog code transducer. As a result, initial data (uninterruptible realization of input $x(t)$ and output $y(t)$ signals) were expressed by the following sequence of discret numbers:

$$
\begin{aligned}
& \left\{x_{1}, x_{2}, x_{3}, \ldots, x_{N-1}, x_{N},\right\}, x_{k}=(k \Delta t), \\
& \left\{y_{1}, y_{2}, y_{3}, \ldots, x_{N-1}, x_{N},\right\}, y_{k}=(k \Delta t),
\end{aligned}
$$

where

$N=T / \Delta t-$ number of discret values in each sequence, $T_{e}$-length of uninterruptible realization (time of experiment) $\Delta t$ - pitch of time discretization.

At first covariational-spectral analysis was performed.

The evaluation of corresponding single covariational and mutually related covariational functions is calculated by these equations:

$$
\begin{aligned}
& \hat{R}_{x x}(i \Delta t)=\frac{1}{N-i} \sum_{k=1}^{N-i}\left(x_{k}-\bar{x}\right)\left(x_{k+i}-\bar{x}\right), \\
& i=0,1,2, \ldots, S_{N}, \\
& \hat{R}_{x y}(i \Delta t)=\frac{1}{N-i} \sum_{k=1}^{N-i}\left(x_{k}-\bar{x}\right)\left(x_{k+i}-\bar{y}\right), \\
& i=0,1,2, \ldots, S_{N},
\end{aligned}
$$

where

$$
\bar{x}=\frac{1}{N} \sum_{j=1}^{N} x_{j}, \quad \bar{y}=\frac{1}{N} \sum_{j=1}^{N} y_{j},
$$

selected average values of corresponding sequences are $i \Delta \mathrm{t}=\tau$.

The evaluation of spectral planes is defined by the equation:

$$
\begin{aligned}
& \hat{S}_{x x}\left(k \Delta f_{N}\right)=2 \Delta t\left[\hat{R}_{x x}(0)+\right. \\
& \left.+2 \sum_{i=1}^{S-1} \varphi(i) \hat{R}_{x x}(i \Delta t) \cos \frac{2 \pi k i \Delta f_{N}}{f_{d}}\right], \\
& k=0,1,2, \ldots, P_{N} ; \quad P_{N} \leq f_{d} / 2 \Delta f_{N},
\end{aligned}
$$

while the evaluation of mutually related plane $S_{x y}\left(j k \Delta f_{n}\right)$ is calculated as follows:

$$
\hat{S}_{x y}\left(j k \Delta f_{N}\right)=\hat{R}_{e} \hat{S}_{x y}\left(k \Delta f_{N}\right)+j J m_{N} \hat{S}_{x y}\left(k \Delta f_{N}\right),(6)
$$

where

$$
\begin{gathered}
R_{e} \hat{S}_{x y}\left(k \Delta f_{N}\right)=2 \Delta t\left\{\begin{array}{l}
\hat{R}_{x y}(0)+2 \sum_{i=1}^{S-1} \varphi(i)\left[\hat{R}_{x y}(i \Delta t)+\right. \\
\left.\left.+R_{x y}(-i \Delta t)\right] \cos \frac{2 \pi k i \Delta f_{N}}{f_{d}}\right]
\end{array}\right\} \\
J m_{n} \hat{S}_{x y^{\prime}}\left(k \Delta f_{N}\right)=4 \Delta t \sum_{i=1}^{S-1} \varphi(i)\left[\hat{R}_{x y^{\prime}}(i \Delta t)-\right. \\
\left.-\hat{R}_{x y^{\prime}}(-i \Delta t)\right] \sin \frac{2 \pi k i \Delta f_{N}}{f_{d}}
\end{gathered}
$$

where

$\Delta f_{N}-$ pitch of discretness with respect to frequency, $f_{d}=1 / \Delta t$ - frequency of discretization of uninterruptible 
signals, $\varphi(i)$ - weight function rectifying covariational functions.

For the evaluation of spectral planes, weight function of Hemming is used:

$$
\begin{aligned}
& \varphi(i)=0,54+0,46 \cos \frac{\pi i}{S_{N}}, \\
& i=0,1,2, \ldots, S_{N}-1
\end{aligned}
$$

for the correlation-spectral analysis it is necessary to calculate are following dependencies:

$$
S_{N}=\frac{1}{b_{e} \Delta t}, \quad N=\frac{S_{N}}{\varepsilon_{r}^{2}}, T_{e}=N \Delta t,
$$

where $b_{e}$ is equivalent possibility for calculation of spectrum,

$T_{e}$ - observation time for uninterruptible signals (time of experiment),

$\varepsilon_{2}^{2}$ - standard errors of evaluation of spectrum plane,

$\Delta t-$ pitch of time discretness, corresponding to conditions of Kotelnikov theorem

$$
\Delta t \leq-\frac{1}{2 f_{c}}
$$

$f_{c}$ - frequency of cross-section of ideal rectangular spectrum (in real conditions $f_{c}=(1,5 \div 5) f_{m}, f_{m}-$ maximum fre quency, probable in the spec-trum of signal).

Consequently, for selected values $\Delta t, b_{e}$ and $E_{2}^{2}$ it is possible to determine the necessary amount of $S$ values of correlative function, variable $\mathrm{N}$ is experimental value of input and output signals and the time of uninterruptible experiment $T_{e}$. And vice versa, if a variable has values $S$ and $N$, then according to (9) accuracy of spectrum analysis relative to be and $\varepsilon_{2}^{2}$ is determined.

The evaluation of mutually relative spectrum plane and spectrum plane of input signal can be used for the evaluation of characteristics of frequency according to Viner-Hopfa equation:

$$
\begin{aligned}
& \hat{S}_{x y}\left(j \omega_{N}\right)=\hat{W}\left(j \omega_{N}\right) S_{x x}\left(\omega_{N}\right), \\
& -\frac{\pi}{\Delta t} \leq \omega_{N}<\frac{\pi}{\Delta t},
\end{aligned}
$$

where $\omega_{N}$ - cyclic frequency.

However direct solution of (11) leads to great errors when $w\left(j \omega_{N}\right)$ is determined, because current problem is referred to as incorrectly built problem. This is why when dynamical properties of clutches are investigated parametrical methods of identification of linear systems are applied:

$$
y_{K}=W\left(z^{-1}\right) x_{k}+H\left(z^{-1}\right) \xi_{k}
$$

for example, model of parametric type where

$$
\begin{aligned}
& W=\left(z^{-1}\right)=\begin{array}{c}
b\left(z^{-1}\right) \\
1+A\left(z^{-1}\right)
\end{array} \\
& H\left(z^{-1}\right)=\frac{1}{\left[1+A\left(z^{-1}\right)\right]\left[1+G\left(z^{-1}\right)\right]} \\
& B\left(z^{-1}\right)=\sum_{L_{N}=0}^{m_{N}} B_{L_{N}} z^{-L_{N}}, \\
& A\left(z^{-1}\right)=\sum_{i=1}^{m_{N}} a_{i} z^{-i}, \\
& G\left(z^{-1}\right)=\sum_{\gamma=1}^{q_{N}} C_{\gamma} z^{-\gamma}
\end{aligned}
$$

polynomals because of shift $z^{-1}$ for one step backwards, $C \gamma$-numerical coefficients, $\xi_{K}$ - sequence of independent values with zero average and finite dispersion.

Mathematical model (12)-(14) has the following interpretation. Investigated value $y_{k}$ of output rotational vibrations consists of two components. The first is completely determined by input rotational vibrations $x_{k}$, while existing mutually relative link is described by function $W(z-1)$. The second component does not depend on input rotational vibrations and influences the output vibrations of all unknown disturbances. It is interpreted as the realization of a random single regressive process, described by a function $H(\mathrm{z}-1)$. Corresponding characteristics $W\left(j \omega_{n}\right)$ and $H\left(j \omega_{n}\right)$ are obtained from 13 using

$$
z^{-1}=\cos i \omega_{N} \Delta t-j \sin i \omega_{N} \Delta t .
$$

In this form the task of the identification of the system consists of the evaluation of unknown parameters:

$$
\begin{aligned}
& a^{T}=\left(a_{1}, a_{2}, \ldots, a_{m}\right), \quad b^{T}=\left(b_{0}, b_{1, \ldots, b_{m}}\right) \\
& c^{T}=\left(c_{1}, c_{2}, \ldots, c_{q_{n}}\right)
\end{aligned}
$$

of investigated input and output sequence, in the order of $m_{n}$, polynoms of function $W\left(z^{1}\right)$, system and order $q_{n}$ polynom function $H\left(z^{-1}\right)$.

It forms a random filter, as well as a model of real system to some extent. In expression (16) T denotes transposing.

When orders $m_{n}$ and $q_{n}$ are fixed, evaluation of parameters $a, b, c$ are from minimal square criteria

$$
Q(a, b, c)=\sum_{k=n+1}^{N}\left\{H^{-1}\left(z^{-1}\right)\left[y_{k}-W\left(z^{-1}\right) x_{k}\right]\right\}^{2} \rightarrow \min _{a, b, c}(17)
$$

where $N>2 m_{n}+q_{n}+n, n=\max \left(m_{n}, q_{n}\right), N$ - number of observations.

Numerical algorithms for the calculation are obtained on the basis of the evaluation of components, leading in the current task to $l_{n}$ pitch of equation: 


$$
\begin{aligned}
& \left(\begin{array}{l}
\hat{a}_{l_{N}} \\
\hat{b}_{l_{N}}
\end{array}\right)=\left(\sum_{k=n+1}^{N} d_{k} d_{k}^{T}\right)^{-1}\left(\sum_{k=n+1}^{N} y_{k}^{1} d_{k}\right) \\
& \hat{c}_{l_{N}}=\left(\sum_{k=n+1}^{N} h_{k} h_{k}^{T}\right)^{-1}\left(\sum_{k=n+1}^{N} v_{k} h_{k}\right), l_{N}=1,2, \ldots \\
& d_{k}^{T}=\left\{y_{y-1}^{\perp}, y_{y-2}^{\perp}, \ldots, y_{k-n_{N}}^{\perp}, x_{k}^{\perp}, x_{k-1}^{\perp}, \ldots, x_{k-n_{N}}^{\perp}\right\} \\
& h_{k}^{T}=\left\{V_{k-1}, V_{k-2}, \ldots, V_{k-q_{N}}\right\},
\end{aligned}
$$

where row-vector of $2 m_{n}+1$ and $q_{n}$ appropriately. Elements which are calculated by recurrence equation

$$
\begin{aligned}
& y_{k}^{\perp}=\left[1+\hat{G}_{l_{N}-1}\left(z^{-1}\right)\right] y_{k} \\
& x_{k}^{\perp}=\left[1+\hat{G}_{l_{N}-1}\left(z^{-1}\right)\right] x_{k} \\
& V_{k}=\left[1+\hat{A}_{l_{N}}\left(z^{-1}\right)\right] y_{k}-\hat{B}_{l_{N}}\left(z^{-1}\right) x_{k}
\end{aligned}
$$

where

$$
\hat{A}_{I_{N}}\left(z^{-1}\right), \hat{B}_{l_{N}}\left(z^{-1}\right), \hat{G}_{l_{N}-1}\left(z^{-1}\right)
$$

polynoms of the shape (14) as $a=\hat{a}_{\mathrm{ln}}, b=\hat{b}_{n}, c=\hat{c}_{\mathrm{ln}-1}$ are substituted.

Structural scheme for the evaluation of parameters is given in Fig 3.

$$
\begin{aligned}
& \eta_{e}=\left(1-\frac{\hat{\sigma}_{e}^{2}}{\hat{\sigma}_{y}^{2}}\right) 100 \% ; \\
& \eta_{\varepsilon}=\left(1-\frac{\hat{\sigma}_{\varepsilon}^{2}}{\hat{\sigma}_{y}^{2}}\right) 100 \%,
\end{aligned}
$$

where $\hat{\sigma}_{e}^{2}$ evaluation of error of sequence dispersion

$$
e_{k}=y_{k}-\hat{W}\left(z^{-1}\right) x_{k},
$$

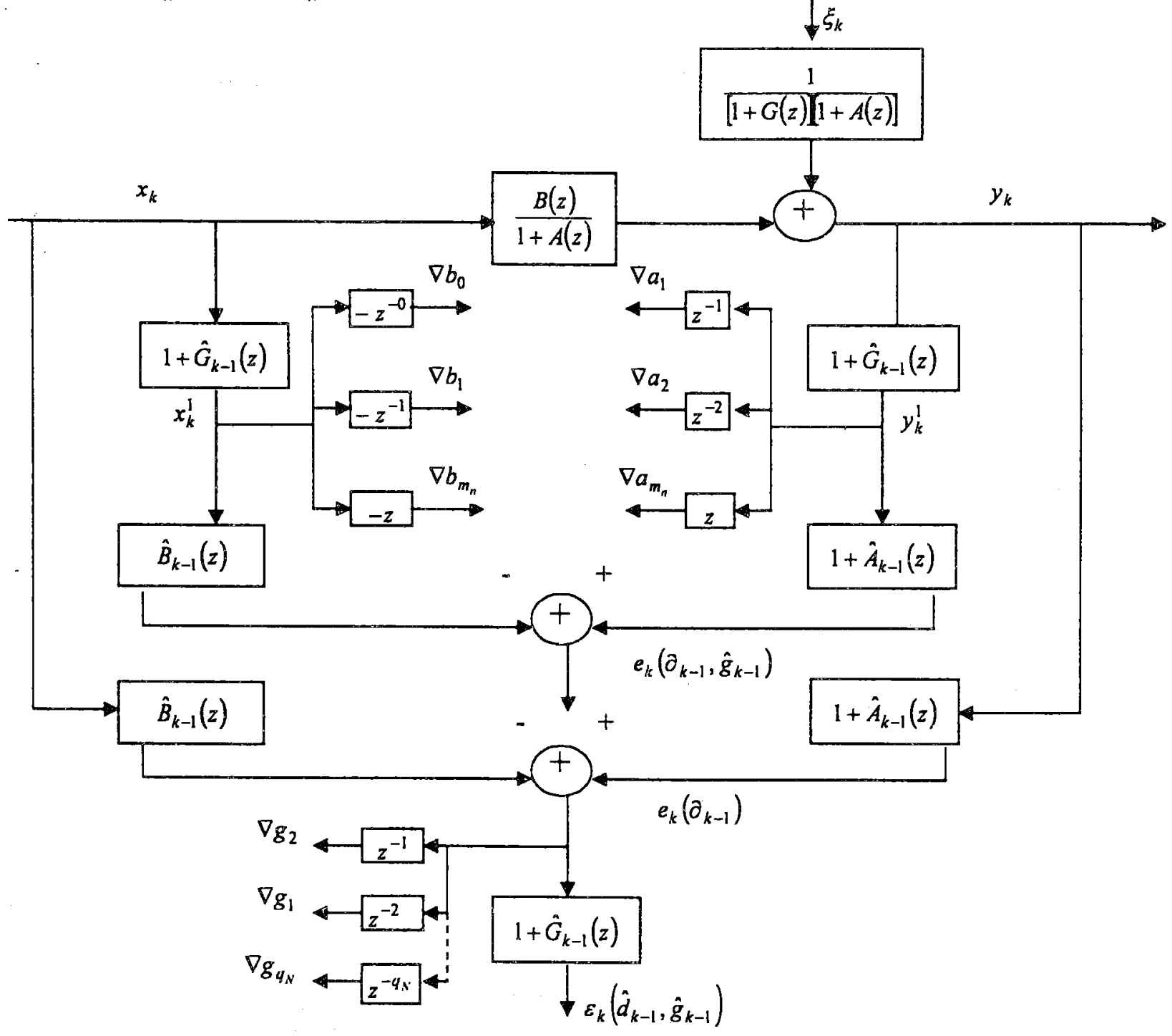

Fig 3. Block - diagram of the estimation of parameters of chosen dynamic model of the system 
$\hat{\sigma}_{\varepsilon}^{2}$ - evaluation of error of sequence dispersion.

$\varepsilon_{k}=\hat{H}^{-1}\left(z^{-1}\right) e_{k}=\hat{H}^{-1}\left(z^{-1}\right)\left[y_{k}-\hat{W}\left(z^{-1}\right) x_{k}\right]$,

$\hat{\sigma}_{y}^{2}$ - evaluation of dispersion of output sequence $y_{k}$, $\hat{W}\left(z^{-1}\right) \cdot \hat{H}\left(z^{-1}\right)$ - appropriate evaluation of transferring functions $\hat{W}\left(z^{-1}\right), H\left(z^{-1}\right)$.

Value $\eta_{e}$ as a pointer in per cent of extent of system "determinacy" with respect to the selected input signal.

$\eta_{e}$ determines that part of output dispersion, which can be only predicted according to input signal $x_{k}$ using

transfer function $W\left(z^{-1}\right)$. By value $\eta_{\varepsilon}$ that part of output dispersion is determined which can be predicted by transfer functions $W\left(z^{-1}\right)$ and $H\left(z^{-1}\right)$.

\section{Results}

When results are evaluated, it is recommended to use only those observations that were not used for locating unknown parameters of the model. Consequently, all the observations fall under two categories: "training" and "examination" selections. The second group tends to be smaller in its size.

Magnitudes of type (21) can be used and for the selection of polynoms corresponding to order (14).

$$
\eta_{e}^{m_{N}+1}-\eta_{e}^{m_{N}}<C_{N}, \eta_{\varepsilon}^{q_{N}+1}-\eta_{\varepsilon}^{q_{N}}<C_{N}
$$

$C_{N}$ - small constant. $N=8000$ discreet values of input and output rotational vibrations for half-coupling. For frequency of discreetness $f_{d}=4,000 \mathrm{~Hz}$ this is equal to time of uninterruptible experiment $T_{e}=2 \mathrm{sec}$. Equivalent allowable ability for spectrum is assumed as $B_{c}=20 \mathrm{~Hz}$, while standard error $\varepsilon_{r}^{2}=0,025$. Dynamical models of rotational vibration ECC are built (Fig 3) in this way: at first $N_{1}=7000$ observations are determined unknown parameters, lastly $N_{2}=1000$ value signals are used for the evaluation. After the determination of $\hat{a}$ and $\hat{b}$ parameters of a model characteristics are calculated according to:

$$
A(k \Delta f)=\hat{W}\left(j k \Delta f_{N}\right)=
$$

$$
\sqrt{\frac{\left(\sum_{i=0}^{m_{N}} \hat{b}_{i} \cos \frac{2 \pi k i \Delta f_{N}}{f_{d}}\right)^{2}+\left(\sum_{i=0}^{m_{N}} \hat{b}_{i} \sin \frac{2 \pi k i \Delta f_{N}}{f_{d}}\right)^{2}}{\left(1+\sum_{i=1}^{m_{N}} \hat{a}_{i} \cos \frac{2 \pi k i \Delta f_{N}}{f_{d}}\right)^{2}+\left(\sum_{i=1}^{m_{N}} \hat{a}_{i} \sin \frac{2 \pi k i \Delta f_{N}}{f_{d}}\right)^{2}}}
$$

where $k=0,1, \ldots P_{N^{*}}$.

Fig 4 illustrates appropriate spectrum rotational analysis of covariative functions, spectrum planes and amplitude-frequency characteristics shows ring type ECC that investigated transfers relatively high extent cohesion between half-couplings in a wide range, being superior to couplings of analog design. Investigating ECC it is pos- sible to come to the conclusion that "centrifugal mass" from the point of view of vibration damping, is a positive factor. This "mass" considerably reduces rotational vibrations, even though it extends the zone of resonance a little when operating at low resonance a little when operating at low frequencies.

\section{Conclusions}

1. In order to obtain more precise measurement results it is necessary to use variable carrier frequency from clock-pulse generator.

2. In order to avoid shutdown and re-calibration of the test-stand it is necessary to use magnetic data media (magnetic disks).

3. In order to scan and write new clock signals precisely it is necessary to use blocks of 5 magnetic heads.

4. In order to obtain continuous deviation of rotary frequency of a driving motor it is necessary to use thyristory power converter with phase control.

5. Carrying out the preset program for dynamic loading of coupling load, the pulse signal of desired shape and amplitude is transmitted via interface from the computer to the load generator-brake

6. Vibrcinsulating properties of the coupling can be evaluated comparing experimental rotary vibrations of driving and driven half - couplings recorded at discrete time moments, $x$ and $y$ accordingly.

7. Processing signals it is necessary to evaluate the established transfer function and its measurement error.

8. Only having determined the transfer function of the coupling, it is possible to compose the amplitude frequency response based on discrete values across the whole range of rotary frequencies under investigation.

\section{References}

1. The USSR Certificate of Invention 1596215. The Universal Unit for Measurement of Dynamic Characteristics of Couplings / L. A. Zubavičius, B. P. Spruogis, V. I. Davulis. Published in 1999. Bull. 36 (in Russian).

2. Spruogis B. Universal dynamic test rig. Transport Engineering (Transporias). Vilnius: Technika, 1995, No 2(11), p $51-59$ (in Lithuanian).

3. Spruogis B. The devices of transmission and stabilization of rotary motion (Устройства для передачи и стабилизацин врапательного движения). Vilnius: Technika, 1997. 476 p (in Russian).

4. Bendat J., Peersol A. Application of Correlation and Spectral Analysis (Применение корреляционного и спектрального анализа). Moscow: Mir, 1983. 312 p (in Russian).

5. Kavolèlis A.-P. Mechanisms and Devices for a Transmission of Rotation, Vibro-Protection and Stabilization on the 
Basis of Centrifugal Forces (Механизмы и устройства для передачи врашения, виброзащиты и стабилизапии на основе центробежных сил). Vilnius, 1983. 186 p (in Russian).

6. Push A. An Investigation of Spindle Jonctions Using the Method of Statistical Simulation. Machines and Instruments (Станки и инструменты). M., 1982, No 1, p 9-12 (in Russian).

7. Vibrations in Engineering: The Reference Book (Вибрации в технике). In the volumes 6 and 5 / Edited D. N. Reshetov Moscow: Mashinostroyeniye, 1979. 389 p (in Russian).

8. The Russian Federation Certificate of Invention 2063007. The Meter of the Moment of Rotation (Бюлетень изобретений). A. N. Shilin. Published in 1996. Bull. 18 (in Russian).

9. The USSR Certificate of Invention. 619811. The Device for Measurement of the Moment of Rotation (Бюлетень изобретений). R. N. Zayev. Published in 1978. Bull. 30 (in Russian).

10. Kochubyevskij I. D. The Loading Systems for Investigations and Tests of Machines and Mechanisms (Системы нагружения для исследования и испытания машины и механизмов). Moscow: Nauka, 1985. 221 p (in Russian).
11. Konarchuk V. E. Longevity and Wear of Engines on Dynamic Modes of Operation (Долговенность и износ двигателей при динамических режимах работы). Kicv: Naukova dumka, 1978. 255 p (in Russian).

12. The Measuring Set for Tests of Hydro-mechanical Drives. Autocar Industry (Авмомобильная промымленноспь). Moscow. 1983, 3, p 35-36 (in Russian).

13. Yamaguchi N., Kobori I., Kobayashi N. Experimental study for a heat transport device using magnetic fluid: improivement by a regenerative thermo magnetic heat cycle. Journal of Mechanical Engineering Science. 2000, Vol, No C7. ISSN 0954-4062, $\mathrm{p}$

14. Tian X. G. and Tao X. M. Torsion Measurement using Fiber Bragg Grating Sensors. Experimental mechanics. An International Journal, 2001, Vol 41, No 3, p 248-253.

15. Kaminskas V., Nemura A. Statistical Methods in an Identification of Dynamic Systems (Статистические методы в идентификации динамических систем). Vilnius: Mintis, 1975. $317 \mathrm{p}$ (in Russian).

16. Vekteris V., Jurevičius M., Cereška A. Methods of Statistical Diagnostics of Rotor Systems. Measurements (Matavimai). Kaunas: Technologija, 2001, No 8, p 31 -34 (in Lithuanian). 